\title{
La contabilidad en la conservación. Atributos del paisaje cultural cafetero inscrito en la lista de la UNESCO como patrimonio mundial de la humanidad. Estudio de caso
}

\author{
Accounting in conservation. Attributes of the inscribed coffee cultural landscape \\ on the UNESCO list as world heritage of humanity. Case study
}

Recibido: 20-06-2018 • Aprobado: 10-10-2018 • Página inicial: 133 - Página final: 145

\author{
Lina Marcela Sánchez Vásquez*
}

\begin{abstract}
Resumen: el artículo parte del postulado según el cual la contabilidad como saber se encuentra inmersa en la escena social. Ante la existencia de realidades no financieras, su forma de incorporar hechos que escapan a la esfera económica requiere el desarrollo de métodos de valoración, reconocimiento y comunicación que sean afines a las mismas denominadas contabilidades emergentes. En el campo de los activos inmateriales, como es el caso del paisaje cultural cafetero, se presenta una propuesta de contabilidad cultural, modelo en construcción que se enfrenta a un desafío de desarrollo de la instrumentalización contable. Se centró en el análisis de los atributos del Paisaje Cultural Cafetero (PCC), fundamentado a su vez en conceptos de la contabilidad cultural y su aporte a la conservación del Patrimonio Cultural. Su inclusión en la lista de patrimonio de la humanidad por parte de la Unesco responde, como campo de estudio, a la inquietud por la corresponsabilidad social que le compete a la contabilidad respecto de la salvaguarda del patrimonio cultural. El Paisaje Cultural Cafetero debe entenderse como el testimonio del modo de vida de unos habitantes y su relación en un entorno determinado, que cumple con unas características culturales y naturales.
\end{abstract}

Palabras clave: paisaje cultural cafetero, contabilidad cultural, valoración, patrimonio cultural.

\begin{abstract}
This article of reflection focused on the analysis of the attributes of the Coffee Cultural Landscape (PCC), based in turn on concepts of cultural accounting and its contribution to the conservation of Cultural Heritage, taking into account the approach that is known what you have and what you are worth, you know what is lost; also argued the theory that the landscape has vulnerable resources, and are directly affected by climate changes, past time, modernity, economic-financial pressures, globalization and other external and internal aspects of the geographical area of the territory. A review was made of the main components that identify the Cultural Landscape, the benefits that the inscription of the Coffee Cultural Landscape brings in the list of world heritage of humanity and specifically those that correspond to the department of Quindío, and having a broad knowledge from the above aspects, raise a process of awareness for the protection and conservation of Cultural Heritage in the department of Quindio, the latter as the final work of the research project. Each one of the topics was exposed in a detailed way, but limiting the information only with the direct relation with the subject of the study, so that a clear understanding of the points that are treated was obtained and to be able to reach the objectives that were established. The Coffee Cultural Landscape should be understood as the testimony of the way of life of some inhabitants and their relationship in a given environment, which meets certain cultural and natural characteristics.
\end{abstract}

Keywords: coffee cultural landscape, cultural accounting, valuation, cultural equity.

JEL: M41 - M49

\footnotetext{
* Estudiante de Doctorado en Administración Gerencial, Magister en Desarrollo sostenible y medio ambiente, Contadora Pública de la Universidad del Quindío. Docente investigadora del Programa de Contaduría Pública de la Universidad Autónoma Latinoamericana de Medellín, Antioquia. Investigadora asociada de Colciencias en el Área ciencias sociales interdisciplinarias. Formadora y asesora en procesos de implementación de Niif en organizaciones del sector público y privado.

lina.sanchezva@unaula.edu.co

Enlace ORCID: https://orcid.org/0000-0003-3911-8367
} 


\section{Comptabilité en conservation Attributs du paysage culturel du café inscrit sur la liste de I'Unesco au patrimoine mondial de I'humanité. Étude de cas}

Résume: l'article part du postulat selon quelle comptabilité en tant que connaissance est immergé dans la scène sociale. Compte tenu de l'existence de réalités non financières sa façon d'incorporer des faits qui échappent à la sphère économique nécessite l'élaboration de méthodes d'évaluation, la reconnaissance et la communication qui sont liées à les mêmes comptes dits émergents. Dans le domaine des actifs incorporels, comme c'est le cas Dans le cas du paysage culturel du café, le une proposition de comptabilité culturelle, modèle en construction qui fait face à un défi de développement de l'instrumentation comptable. Il axé sur l'analyse des attributs du paysage Café culturel (PCC), basé à son tour sur concepts de comptabilité culturelle et leur contribution à la Conservation $\mathrm{du}$ patrimoine culturel. Son inclusion sur la liste du patrimoine mondial par l'Unesco répond, en tant que domaine d'étude, à l'inquiétude pour la coresponsabilité sociale il appartient à la comptabilité en ce qui concerne la sauvegarde du patrimoine culturel. Le paysage culturel du café doit être compris comme le témoignage du mode de la vie de certains habitants et leur relation dans un environnement déterminé, qui répond à certaines caractéristiques culturel et naturel.

Mots-clés: café paysage culturel, comptabilité culturel, valorisation, patrimoine culturel.

\section{Contabilidade em conservação Atributos da paisagem cultural do café inscrito na lista da Unesco como Património Mundial da humanidade. Estudo de caso}

Resumo: o artigo parte do postulado de acordo com que contabilidade como conhecimento é imerso na cena social. Dada a existência de realidades não financeiras a sua maneira de incorporar fatos que escapam à esfera econômica requer o desenvolvimento de métodos de avaliação, reconhecimento e comunicação que estão relacionados com as mesmas chamadas contas emergentes. No campo dos ativos intangíveis, como é No caso da paisagem cultural do café, a uma proposta de contabilidade cultural, modelo em construção que enfrenta um desafio de desenvolvimento de instrumentação contábil. Ele focado na análise dos atributos da Paisagem Café Cultural (PCC), baseado por sua vez conceitos de contabilidade cultural e sua contribuição para o Conservação do Patrimônio Cultural. Sua inclusão na lista do património mundial por da Unesco responde, como campo de estudo, a a inquietude pela co-responsabilidade social que cabe à contabilidade sobre a salvaguarda património cultural. A paisagem cultural do café deve ser entendido como o testemunho do modo de vida de alguns habitantes e sua relação em um ambiente determinado, que atende certas características cultural e natural

Palavras-chave: paisagem cultural do café, contabilidade cultural, valorização, herança cultural. 


\section{Introducción}

Los bienes de interés cultural han adquirido especial relevancia en el contexto internacional y nacional en función de su valor social como testigos de la historia y preservadores de la cultura. En el departamento del Quindío esta realidad se hace cercana y latente en especial a partir del año 2011, cuando la Unesco incluyó el Paisaje Cultural Cafetero en la lista del patrimonio inmaterial de la Humanidad, incorporando con ello una connotación de interés general sobre el territorio a la vez que supone la necesidad político-institucional por preservarlo y conservarlo.

En el Paisaje Cultural Cafetero existen algunas amenazas que apuntan a la destrucción de los atributos naturales, culturales y sociales, fundamentales para la continuidad de su inscripción en la lista de Patrimonio Mundial de la Humanidad de la Unesco, pero también esenciales para el desarrollo de la persona humana que habita en este territorio.

En cuanto a amenazas, el departamento del Quindío no es una excepción, las amenazas ambientales: la contaminación de los recursos naturales es la más común, de las fuentes de agua, con la pérdida de microcuencas, prácticas agrícolas con usos inadecuados de agroquímicos y pérdida de la biodiversidad; y algunos riesgos culturales: el desinterés, el desconocimiento y la falta de pertenencia por aquellas características cafeteras que nos identifican.

Contablemente existe una responsabilidad frente a este campo del saber en la escena social, que consiste en la capacidad para identificar, medir, clasificar, informar, es decir, preservar el patrimonio. Regularmente, estas acciones han sido abanderadas desde la perspectiva financiera, por ser la esfera económica el principal frente de uso y apropiación contable. No obstante, lo cultural tiene una visión diferente, situada en el campo de lo social, mediada por valores, percepciones y acuerdos colectivos difícilmente cuantificables a través de lo financiero.

De lo anterior se colige la necesidad de interrogar por el uso y la responsabilidad que le corresponden a la contabilidad como campo del saber en la conservación y preservación del paisaje cultural cafetero, asunto que se desarrolla en este artículo.

\section{Metodología}

Los métodos que se usaron en el desarrollo del artículo fueron el exhaustivo rastreo bibliográfico en libros y revistas especializadas de la normativa y 


\section{Contexto Sanchere, L.}

estudios sobre patrimonio cultural. De igual manera, como insumo fundamental para su reconocimiento contable, se abordó la búsqueda sobre artículos y publicaciones científicas, así como estudios sectoriales enfocados en el paisaje cultural cafetero. Se parte de la Declaración de la Unesco de 2011, que convierte una porción del territorio del eje cafetero en Patrimonio Cultural de la Humanidad, y termina en un análisis de los atributos del Paisaje Cultural Cafetero, específicamente en el departamento del Quindío. Se emplea un enfoque deductivo, pasando entonces de lo general a lo particular, sin perder en ningún momento el enfoque de análisis global que requiere la investigación, y se analizan las realidades culturales que pueden ser informadas de manera financiera y no financiera.

\section{Discusión de referentes}

Son cuatro los valores que determinan la excepcionalidad del Paisaje Cultural Cafetero (PCC) y son la base de su postulación como patrimonio mundial:

1. Trabajo familiar, generacional e histórico para la producción de un café de excelente calidad, en el marco de un desarrollo sostenible.

2. Cultura cafetera para el mundo.

3. Capital social estratégico construido alrededor de una institucionalidad.

4. Relación entre tradición y tecnología para garantizar la calidad y sostenibilidad del producto.

Desde el año 2001 el Estado colombiano, en su compromiso de vincular las políticas sociales y económicas con el campo cultural, ha promovido un proceso de valoración y reconocimiento del territorio cafetero con el fin de postular su inscripción ante la Unesco en la lista del patrimonio mundial.

\section{Resultados}

\section{Contabilidad y cultura}

Para Fowler (2005), sólo deben computarse como activos los bienes intangibles para los que exista la posibilidad de medición objetiva, situación que se presenta cuando su incorporación al activo implica un costo perfectamente identificable, como ocurre cuando se procede a su adquisición. No podrán incluirse en el activo los bienes intangibles inseparables del conjunto del organismo y los cuales éste desarrolla. Esa postura teórica se ha convertido en un obstáculo para efectos de la posibilidad de incorporar los bienes culturales (en el caso de manifestaciones culturales) y la subjetividad que acompaña al proceso de valoración, atado a la percepción social del beneficio reportado. 
Abordar el concepto de cultura, al igual que el de contabilidad, nos pone ante un abanico de posibilidades conceptuales. La cultura posee múltiples sentidos, teorías, y escuelas de pensamiento. Según el diccionario filosófico de Abbagnano (1961), el término "cultura" tiene dos significados fundamentales: el primero concerniente a "Formación del hombre, su mejoramiento y perfeccionamiento", el segundo hace alusión al resultado de esta formación, como un "conjunto de los modos de vivir y de pensar cultivados, civilizados, pulimentados a los que se suele dar el nombre de civilización".

En su libro La cultura: versiones y definiciones, Warley plantea que el concepto de cultura presenta dicotomías porque, por un lado, puede considerarse concreto, particular, individual, homogéneo, una serie de cosas, bienes materiales y catalogables, libros, películas, pinturas, etc., aquello que singulariza a una nación, una comunidad o un sector social determinado; también se puede hablar de una cultura individual al referirse a los hábitos, conductas, gustos, moralidad de un sujeto; en fin, la cultura es un bloque sólido que se vuelve característico precisamente por su unicidad, pero, por otra parte, la cultura puede considerarse abstracta, universal, social, heterogénea, un conjunto de valores, hábitos y conductas concebidos en relación con normas y pautas de obvia naturaleza social, una característica de la especie humana, es una mezcla de múltiples tradiciones (Warley, 2003).

Empero, tras las múltiples dicotomías del concepto se encuentran conjuntos de manifestaciones -económica, sociopolítica y ambiental- que se pueden abordar como dimensiones de la cultura. En el texto "Algunas relaciones entre contabilidad y cultura" (Ocampo, Lastra y Gómez, 2008), los autores expresan (como resultado de su investigación) que la cultura tiene una perspectiva económica cuando se encuentran elementos como mercancía, acumulación y consumo, es así como las industrias culturales promueven obras de arte, museos, sitios de turismo y bibliotecas para obtener beneficios económicos.

No obstante, las alternativas de reconocimiento contable se establecen al comprender la injerencia que los bienes carentes de sustancia material, denominados intangibles, tienen en el desarrollo tanto de los territorios como de los organismos económicos. El modelo financiero internacional contempla la inclusión de intangibles, bajo el criterio de considerarlos independientes de los demás activos de la organización, que generen beneficio económico futuro y puedan medirse con fiabilidad. La existencia de estos criterios en el nivel organizacional privado y de un régimen de contabilidad pública para patrimonio cultural, son los mecanismos de lo contable para hacer frente a la necesidad de reconocer e incluir los activos intangibles. 


\section{Contexto sincronz L}

En lo referente a bienes culturales, el régimen de contabilidad pública establece todos los parámetros necesarios para contabilizar el patrimonio cultural, incluso determina el catálogo de cuentas donde deben estar registrados, pero no contempla la forma de valuación de dichos bienes para que de esta forma se pueda realizar una adecuada contabilización. Este marco de contabilidad pública permite que la entidad no realice el registro contable como debe ser, lo que genera mayores riesgos de que el bien de patrimonio cultural se pierda o sufra daño, porque no se tiene registrado contablemente, lo cual impide un posible comodato para su conservación.

En consonancia con lo anterior, el Manual para inventarios de bienes culturales inmuebles (Mincultura, 2005) constituye una guía para el levantamiento de características inventariables. Este documento se convierte en línea base de la política relacionada con el patrimonio cultural y apoya el fortalecimiento de instituciones territoriales para el manejo del patrimonio. Cuando una colectividad reconoce que el patrimonio es la expresión de su cultura, establece el principio de corresponsabilidad entre la comunidad y las autoridades para su manejo, protección y conservación.

\section{El Paisaje Cultural Cafetero (PCC)}

Según la Unesco, por paisaje cultural se entiende el resultado de las actividades humanas en un territorio concreto. Los componentes que identifican el paisaje cultural se esquematizan en la Figura 1.

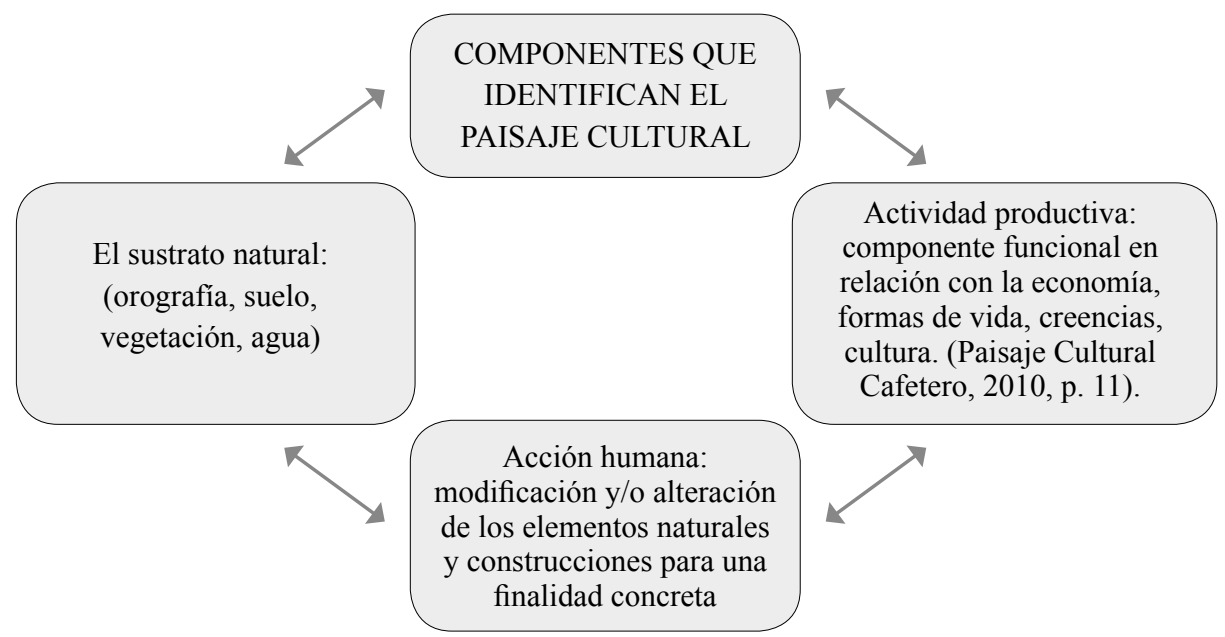

Figura 1. Componentes del paisaje cultural.

Elaboración propia, 2018. 
Los cuatro valores que son la base de la postulación del PCC como patrimonio mundial son los siguientes:

- Esfuerzo humano, familiar, generacional e histórico para la producción de un café de excelente calidad en el marco de un desarrollo humano sostenible: más de cien años de adaptación del cultivo del café, es el resultado del esfuerzo de los pobladores que llegaron a estas tierras y que encontraron en el café una fuente para su sustento. En la actualidad la caficultura se encuentra sobre las laderas de las cadenas montañosas, por esto recibe el nombre de café de montaña.

- Cultura cafetera para el mundo: la cultura de esta región está ligada a la colonización antioqueña, buscando ser propietarios de tierras, la minería pierde importancia y el café se convirtió en una buena opción para hacer uso de las tierras, es así, como la caficultura llegó a ser la base de la economía de la región, y a su alrededor se desarrollaron una serie de manifestaciones que se han transmitido a todas las generaciones. Algunas de las manifestaciones que definen la cultura cafetera para el mundo se numeran en la Tabla 1.

Tabla 1.

Manifestaciones que definen la cultura cafetera

\begin{tabular}{ll}
\hline Personajes y objetos & \\
\hline El arriero & Ayudó a abrir caminos junto con sus mulas y bueyes. \\
\hline El hacha y el machete & $\begin{array}{l}\text { Primero se utilizaron para abrir camino y más adelante para } \\
\text { el deshierbe en los cultivos. }\end{array}$ \\
\hline
\end{tabular}

El jeep Willys

Medio de transporte utilizado para llevar cargas de café de la finca hasta los almacenes de venta, también se utiliza como transporte público en las zonas rurales, y en coroteos (mudanzas).

\begin{tabular}{ll}
\hline Juan Valdez & Personaje que identifica a los cafeteros colombianos ante el mundo. \\
\hline Mitos o leyendas & $\begin{array}{l}\text { Tales como la Madremonte, la Patasola, entre otros, que, de } \\
\text { acuerdo con la tradición oral, viven en el monte y aparecen cuando } \\
\text { son provocados. Son creaciones de la imaginación de las personas } \\
\text { que vinieron de Antioquia, reconocidas como paisas. }\end{array}$ \\
\hline Saberes culinarios & $\begin{array}{l}\text { Representados por su cantidad, modos de preparación, } \\
\text { presentación y colorido. }\end{array}$ \\
\hline Vestuario & $\begin{array}{l}\text { Representado por el arriero y su atuendo que es el poncho o ruana } \\
\text { el sombrero, el carriel, aun caficultores utilizan este vestuario. }\end{array}$ \\
\hline
\end{tabular}




\section{Contexto sinconz L}

Personajes y objetos

Fiestas y festivales

Varios están asociados con la cultura cafetera, como las fiestas de Calarcá, las fiestas del Canasto.

Los colonos antioqueños trajeron a esta región el modelo de la arquitectura que era de influencia española. En su necesidad de fundar nuevos pueblos se expandieron hacia el sur para colonizar más territorio, cuando la topografía no permitía seguir el diseño español trazaban en una calle la plaza, la iglesia y el cabildo, luego procedían a repartir los solares para los colonos.

En las poblaciones localizadas en la zona del PCC, pese a que la topografía a veces no permitía construir como lo deseaban, lograron de manera obstinada mantener los trazados para todos los pueblos, que todos tuvieran la plaza y el templo como emblema de cada uno de ellos. En los filos de las montañas empiezan a construir viviendas, de bahareque y tejas de barro, las puertas y ventanas eran de madera adornadas con calados, los patios y corredores formaban una tipología (en "U", "L", "I" y "O"). Las

La vivienda casas eran adornadas con flores y pájaros, así las casas se convertían en "miradores", con sus dos espacios principales, uno de ellos destinado a la vivienda y el otro destinado a la helda, o sea para el secado del café, también se incluían establos, gallineros, graneros y depósitos. En las fincas más extensas se encuentran habitaciones y baños para los recolectores que llegan a trabajar durante la cosecha de café.

Le dieron un gran uso a la guadua utilizada en la construcción. Las viviendas en guadua le añaden un valor cultural al paisaje cultivado que caracteriza al PCC. También se encuentran en estas poblaciones cementerios que han sido declarados bienes de interés cultural del ámbito nacional, como los de Salamina, Marsella y Circasia. Algunas instituciones, como La Casa de la Cultura de Marsella, el Centro Cultural de Calarcá que se encuentran ubicadas en donde antes estas viviendas pertenecían a colegios, o a familias prestantes de la época.

Elaboración propia, 2018. 
Lo expresado previamente cuenta la historia sobre las tradiciones y formas de vida y de habitar el territorio, aspectos que contribuyeron a la inclusión del PCC en la lista del patrimonio inmaterial de la Unesco: "Son las huellas que han dejado los pobladores en el paisaje de gran parte de los departamentos del Quindío, Risaralda, Caldas y norte del Valle del Cauca y está representado en 15 atributos que lo hacen excepcional" (PCC, 2010, p.13) (ver Tabla 2).

Tabla 2.

Atributos del Paisaje Cultural Cafetero

1. Café de montaña

2. Predominio de café

3. Cultivo en ladera

4. Edad de la caficultura

5. Influencia de la modernización

6. Institucionalidad cafetera y redes económicas afines

7. Tradición histórica en la producción de café

8. Estructura de pequeña propiedad cafetera

9. Cultivos múltiples

10. Tecnologías y formas de producción sostenibles en la cadena productiva del café

11. Patrimonio arquitectónico

12. Patrimonio urbanístico

13. Patrimonio arqueológico

14. Patrimonio natural

15. Disponibilidad hídrica

Tomado de Paisaje Cultural Cafetero (2010, pp.13-16).

En consideración a la vulnerabilidad del PCC y los compromisos inherentes a la declaratoria por parte de la Unesco, se decide elaborar un plan de manejo:

Un documento que expresa cómo se actúa sobre un territorio, de acuerdo a sus posibilidades productivas ambientales, sociales y culturales, establece las acciones que requiere para prevenir, mitigar, controlar, compensar y corregir los posibles efectos o impactos negativos y cómo aprovechar los positivos, causados en desarrollo de un proyecto, obra o actividad (PCC, 2010, p.24).

El PCC es un paisaje vivo y dinámico, por esto su plan de manejo debe establecer las condiciones de conservación y apropiación por parte de los 


\section{Contexto Sanchere, L.}

habitantes, de acuerdo a las actividades económicas que se desarrollan en la región. Los criterios en relación con el sujeto y con el contexto sociocultural se toman como referencias colectivas de identidad, atados a lazos de memoria, significado o apropiación, que aluden a valores simbólicos.

La Ley 397 de 1997 establece que el Estado debe registrar el patrimonio cultural, y además dar los medios necesarios para recuperar los bienes culturales que estén en posesión de terceros. En el año 2007, la Contaduría General de la Nación, mediante la Resolución 354, emitió el Régimen de Contabilidad para el sector público en Colombia, y en virtud de esta ley todos los hechos económicos del sector público deben quedar debidamente registrados, y por esta razón se deben reconocer y medir para posteriormente revelarlos en los estados contables. Por su parte el documento CONPES 3803 de 2014, tiene como objetivo formular una política específica para el PCC con el fin de garantizar la preservación de su valor universal excepcional y mejorar las condiciones para la sostenibilidad ambiental, cultural, social y económica del territorio.

La garantía del inventario se fundamenta en el soporte para realizar el registro del bien como de interés cultural. Así, el valor cultural del bien permite, en términos de patrimonio, que pueda ser protegido mediante una declaratoria como bien de interés cultural en el marco de la Ley 397 de 1997. El manual define las etapas (seis en total) para hacer el inventario y entrega las guías metodológicas y fichas para su realización.

La existencia de bases jurídicas para la protección del patrimonio cultural en el territorio colombiano y su coherencia con las convenciones y acuerdos internacionales son generadores de confianza pública, como herramientas que desde la política pública incorporan los propósitos sociales y gubernamentales para la conservación y salvaguarda del patrimonio.

En el caso del paisaje cultural cafetero, su consideración en las agendas de desarrollo nacional y departamental, entrega mecanismos útiles a nivel social para su seguimiento y control.

Como aporte de la cultura, el patrimonio está directamente relacionado con aquellos elementos y fenómenos que son producto de la interacción social: su producción material y su creación simbólica (la memoria). El resultado de la actividad cultural se manifiesta en presencias tangibles o intangibles, en objetos y en ideas. Esta situación se convierte en un interesante y retador contexto de análisis para la contabilidad. La coexistencia de dimensiones económicas atadas 
a lo financiero y de la supervivencia por siglos del patrimonio cultural, ignorado en lo contable, pareciera ser evidencia de que no era necesaria la participación de lo contable como veedor al servicio del patrimonio cultural. No obstante, en este contexto también es clara la necesidad de desarrollar métodos y formas de medición y valoración para el reconocimiento de las dimensiones de cultura que caben en el campo del discurso contable.

Con referencia a los bienes culturales, Maestromey y Wilches (2007) expresan que, en términos contables, constituyen la base patrimonial que posee toda sociedad y sobre la que se asientan las demás estrategias de gestión cultural, destinadas a la conservación, difusión, tutela, investigación, etc., de dichos bienes, es decir, de aquellos elementos susceptibles de ser incorporados a través del campo contable por su relevancia social y prevalencia como interés general.

No obstante, en la contabilidad financiera tradicional se presentan relaciones de poder y dominación sobre recursos financieros, cuyo objetivo es el lucro de los grupos de interés, específicamente los proveedores de capital. Por tanto surge la imperiosa necesidad de generar una forma de modelización y sistematización alterna a la financiera, a lo cual subyace la propuesta de contabilidades emergentes como contraposición a la respuesta contable centrada en la esfera económica, que desconoce las afectaciones sobre el patrimonio social, en este caso, el correspondiente al patrimonio cultural.

\section{Conclusiones}

Colombia ha desarrollado un importante acervo normativo que promueve la preservación y conservación de patrimonio cultural, por tanto, para efectos de comprender el rol que corresponde a lo contable y cuáles son los bienes culturales que se convierten en objeto de reconocimiento contable, es necesario indicar el alcance normativo de esta intencionalidad .

En este escenario, el reto y a la vez la oportunidad para el saber contable es asumir su rol como garante de la confianza pública mediante el desarrollo de mecanismos que de manera satisfactoria acojan en su seno las realidades culturales para procurar la conservación y permanencia del patrimonio cultural en el tiempo, habida cuenta de la complejidad y multidimensionalidad que encierra lo cultural. La contabilidad cultural, como respuesta a este llamamiento social, viene desarrollando los conceptos y mecanismos a través de los cuales se haga posible el tránsito de lo evidenciado socialmente a lo informado contablemente. 


\section{Contexto Sanchere, L.}

En este punto, lo contable se enfrenta con una limitante, la cual consiste en qué tipo de capital se mantiene y para quién, puesto que al tratarse de bienes de interés cultural, las formas de medición y comunicación escapan a la intencionalidad de rentabilidad para ponerlos en la intención de existencia y conservación. Por tanto, no es posible incluirlos o reconocerlos contablemente en función de su uso o capacidad de generación de beneficio económico, sino atados a la capacidad que tienen de conservar la memoria y la historia social y convertirse en testigos de la evolución o involución social.

En lo referente a las características propias del paisaje cultural cafetero, los rasgos distintivos y las características incluidos en la declaratoria, en razón a su clara y explícita indicación, se convierten en la brújula y banco de sentido para la contabilidad en el departamento de Quindío, no como puerto último sino como dirección en la cual desde su campo del conocimiento deberá dar respuesta. Sólo a través de la construcción de nuevas y mejores formas de identificación, medición, presentación y revelación de las realidades culturales será posible cumplir la promesa de cuidadores del patrimonio cultural.

\section{Referencias}

Abbagnano, N. (1961). Diccionario de Filosofía. México: FCE.

Congreso de la República de Colombia. (1997). Ley 397 de 1997, "Por la cual se desarrollan los artículos 70, 71 y 72 y demás artículos concordantes de la Constitución Política y se dictan normas sobre patrimonio cultural, fomentos y estímulos a la cultura, se crea el Ministerio de la Cultura y se trasladan algunas dependencias". Diario Oficial, 43.102.

Contaduría General de la Nación de Colombia. (2007). Resolución 354, "Por la cual se adopta el régimen de Contabilidad pública, se establece su conformación y se define el ámbito de aplicación".

Departamento Nacional de Planeación de Colombia, Consejo Nacional de Política Económica y Social (Conpes). (2014). Conpes 3803. Política para la preservación del paisaje cultural cafetero de Colombia. Bogotá: DNP.

Fowler, N. (2005). Cuestiones contables fundamentales (4. ${ }^{\mathrm{a}}$ ed.). Buenos Aires: La Ley.

Maestromey, M. y Wilches, S. (6-9 de noviembre de 2007). Los recursos culturales bajo la óptica contable. Jornadas Universitarias de Contabilidad. La Plata, Argentina: s/e. 
Ministerio de Cultura de Colombia (Mincultura). (2005). Manual para Inventarios de Bienes Culturales Inmuebles. Bogotá: Imprenta Nacional.

Ministerio de Cultura de Colombia (Mincultura). (2011). Paisaje cultural cafetero, un paisaje cultural productivo en permanente desarrollo. Bogotá: Nomos impresores.

Ocampo, C., Lastra, C. y Gómez, J. (2008). Algunas relaciones entre contabilidad y cultura. Contaduría, 52. Universidad de Antioquia.

Paisaje Cultural Cafetero (PCC). (2010). Paisaje Cultural Cafetero Colombiano. 3. ${ }^{a}$ ed. Pereira, Risaralda, Colombia.

Rincón, C. (2010). Memorias Taller Técnico. El Paisaje Cultural Cafetero, Cátedra UNESCO, Gestión integral del Patrimonio. Manizales: Artes Gráficas Tizan Ltda.

Unesco. (2011). El Comité del Patrimonio Mundial inscribe cinco sitios más (de Colombia, Sudán, Jordania, Italia y Alemania) en la Lista del Patrimonio Mundial. 35 a reunión del Comité del Patrimonio Mundial. Recuperado de http://www.unesco.org/new/es/media-services/35th-session-of-theworld-heritage-committee/

Warley, G. (2003). La cultura: versiones y definiciones. Buenos Aires: Biblos.

\section{Para citar este artículo:}

Sánchez, L. (2019). La contabilidad en la conservación. Atributos del paisaje cultural cafetero inscrito en la lista de la UNESCO como patrimonio mundial de la humanidad. Estudio de caso. EnContexto, 7(10), 133-145. 